Published in final edited form as:

AIDS. 2017 January 28; 31(3): 437-441. doi:10.1097/QAD.0000000000001316.

\title{
Association of Cognitive Activity and Neurocognitive Function in Blacks and Whites with HIV
}

\author{
Kristin R. KRUEGER ${ }^{1}$, Oluwatoyian ADEYEMI ${ }^{1}$, Sue LEURGANS ${ }^{2,3,4}$, Raj C. SHAH ${ }^{2,5}$, \\ Antonio D. JIMENEZ ${ }^{6}$, Lawrence OUELLET ${ }^{6}$, Alan L. LANDAY ${ }^{7}$, David A. BENNETT ${ }^{2,3}$, and \\ Lisa L. BARNES $2,3,8$ \\ ${ }^{1}$ Cook County Health and Hospital System \\ ${ }^{2}$ Rush Alzheimer's Disease Center, Rush University Medical Center \\ ${ }^{3}$ Department of Neurological Sciences, Rush University Medical Center \\ ${ }^{4}$ Department of Preventive Medicine, Rush University Medical Center \\ ${ }^{5}$ Department of Family Medicine, Rush University Medical Center \\ ${ }^{6}$ Division of Epidemiology and Biostatistics, School of Public Health, University of Illinois Chicago, \\ Chicago, IL USA \\ ${ }^{7}$ Department of Immunology/Microbiology, Rush University Medical Center \\ ${ }^{8}$ Department of Behavioral Sciences, Rush University Medical Center
}

\begin{abstract}
Objective-Older persons with HIV are at risk for impaired cognition, yet there is limited information on modifiable factors associated with neurocognitive function in this group.
Design-This is a cross-sectional observational study of cognitive activities and neurocognitive function.

Methods-We examined the relation between frequency of cognitive activity and current neurocognitive performance in 176 older persons with HIV (70\% African American, 76\% male; mean age $=58.7(\mathrm{SD}=5.5)$; mean education $=13.2(\mathrm{SD}=2.8)$.

\begin{abstract}
Results-In linear regression models adjusted for demographic variables, we found that higher frequency of cognitive activity was associated with better cognition in global cognition, semantic memory, and perceptual speed. Subsequent models that examined the role of race, demonstrated that the association was significant only among Blacks for global cognition, episodic memory, working memory, and perceptual speed (interaction of cognitive activity by race Estimates range $=$ $0.38-0.55$; all $P<0.05)$.
\end{abstract}

Corresponding author: Kristin Krueger, Xkris2blink@gmail.com; (312) 343-8660, 122 W. Central Blvd., Villa Park, IL 60181. Alan L. Landay is on the Scientific Advisory Board for Merck. Lisa L. Barnes is the recipient of grants mentioned above and has no other conflicts of interest. For the remaining authors no conflict of interests or funding sources have been declared.

Author contributions: KRK commissioned the statistical analyses and drafted the manuscript. SL performed the statistical analyses. LLB supervised the data analysis and interpretation. All authors critically reviewed the manuscript and approved the final draft. 
Conclusion-Greater frequency of cognitive activity is associated with better neurocognitive function in older persons with HIV, particularly older Blacks. Longitudinal studies are needed to assess the relation of cognitive activity to change in neurocognitive function in older persons with HIV.

\section{Keywords}

HIV/AIDS; older adults; cognitive activity; neurocognitive function; health disparities

\section{Introduction}

Lifestyle factors can be modified to reduce one's risk of neurocognitive impairment associated with aging [1-4]. Identifying these protective factors among older HIV+ individuals has become critically important, particularly for those at higher risk for ageassociated neurocognitive decline such as Blacks, a population also disproportionately affected by HIV [5].

The literature on aging has consistently shown that cognitive activity, or engaging in mentally stimulating tasks, is protective against neurocognitive decline [1, 6-7]. The few available studies of cognitive activity associated with neurocognitive functioning among older HIV+ individuals [8-10] report promising results, but these studies were limited by small sample size [8], restriction to a single cognitive activity (e.g. internet based stimulation) [9], and indirect or brief measures of cognitive activity [8-10].

In the present study we investigated the frequency of cognitive activity and its association with current neurocognitive function among a group of older HIV+ individuals. Our sample was predominantly Black, which enabled us to explore possible racial differences in this association.

\section{Methods}

\section{Participants}

Participants were 123 Black and 53 White, HIV-seropositive, primarily urban-dwelling men and women age 50 and older, enrolled in a longitudinal study of HIV and aging conducted by the NIMHD-funded Rush Center of Excellence on Disparities in HIV and Aging (CEDHA) at Rush Alzheimer's Disease Center in Chicago. The study protocol was approved by the IRBs in the study's catchment area (Rush University Medical Center, University of Illinois at Chicago, and the Cook County Health and Hospitals System). All participants provided written informed consent.

Table 1 shows participant characteristics. All participants were free of AIDS-defining or other neurologic disorders, with no history of substance use for a minimum of three months. All participants were on combination antiretroviral therapy (CART) with a mean CD4 count of 621 cells/mm3 and 167 (95\%) participants had undetectable HIVNA. All participants undergo annual evaluations that include medical history, neurocognitive and motor function testing, and assessment of risk factors for neurocognitive decline. 


\section{Materials and Procedure}

Cognitive Activity-Participants completed a validated 9-item questionnaire [7] that assessed frequency of cognitive activities such as reading newspapers, magazines, or books; visiting museums or libraries; and attending a concert or play. Participants were asked to rate their participation frequency on a 5-point Likert scale ranging from once or less per year (1) to almost every day (5). The final cognitive activity score consists of the mean of all nine items.

Neurocognitive Function-Participants completed a battery of 19 standardized neurocognitive tests that measured five neurocognitive domains: episodic memory, semantic memory, working memory, perceptual speed, and visuospatial ability. This battery has been used in multiple studies of aging and described in detail previously [1]. In particular, composite scores for the five neurocognitive domains were created by converting raw scores of the individual tests into $z$ scores, and averaging the $z$ scores. An index of global cognition was computed by averaging all of the individual $z$ scores [1].

Covariates-Age was determined from date of birth and date of enrollment; and education was determined as the number of years of formal education completed. Race and ethnicity were determined with questions from the 2000 US Census. Given the possible association with cognition, we included additional covariates in our analyses, in separate models. These included nadir CD4 count, hepatitis C virus (HCV) serostatus, and index of past illicit drug (substance) use derived from the self-reported lifetime number of different drug types used (e.g., cannabis, opioids, amphetamines, etc). Participants' lifetime CD4 nadir and diagnosis of Hepatitis $\mathrm{C}$ were extracted from their medical chart using the clinic visit closest in time to the neurocognitive evaluation. Eight medical conditions were identified in at least $10 \%$ of study participants based on self-report: hypertension, hypercholesterolemia, shingles, lung disease, neuropathy, liver disease, and poor circulation in the legs and feet. A vascular risk summary score analogous to Framingham Study indices [11] was also calculated based on history of diabetes mellitus, hypertension, and smoking (scored 0-3) as previously described by our group [12]. Depressive symptoms were assessed using a modified version of the Center for Epidemiological Studies Depression (CES-D) scale [13].

Data Analyses-We compared the average frequency of each of the nine cognitive activities for Blacks and Whites, with $t$-tests. We then examined the cross-sectional relationship of the cognitive activity and neurocognitive test scores using multiple linear regression models adjusting for age, sex, education, and race, and adding other covariates (CD4 nadir, depressive symptoms, average number of drugs used in a lifetime, number of vascular risk factors and number of medical conditions), one at a time to existing models. In order to examine whether the association between race and frequency of cognitive activity was comparable in Blacks and Whites, we added a term for the interaction between race and frequency of cognitive activity. A priori level of statistical significance was 0.05 .

Programming was done in SAS PROC NLMIX [14]. All models were examined graphically and analytically, with assumptions judged to be adequately met. 


\section{Results}

\section{Descriptive Characteristics of Participants}

The majority of the $176 \mathrm{HIV}+$ participants was Black (70\%), male (76\%), with an average age of 58.7 years $(\mathrm{SD}=5.5)$, and an average of 13.2 years of formal education $(\mathrm{SD}=2.8)$.

Years of education was significantly higher among Whites compared to Blacks $(\mathrm{t}=4.4) P<$ 0.01 ). Whites also reported incomes of $<\$ 25,000$ less frequently compared to Blacks (49\% vs. $\left.81 \% ; \chi^{2}=36.01 ; p<0.001\right)$. Blacks and Whites were generally comparable on other demographic variables measured, including CD4 nadir, medical conditions, vascular risk score, depressive symptoms, and MMSE score. There were no racial differences in percent virally suppressed or in the number on CART. See Table 1 for complete descriptive comparisons between Blacks and Whites.

\section{Cognitive Activity}

Compared with Whites, Blacks reported less frequent participation in three of the nine cognitive activities measured: reading per day, visiting a museum and attending concerts. There were no significant differences in the other activities queried; however, there was a trend for Blacks to report spending more time playing games compared to Whites.

\section{Cognitive Activity Frequency and Demographic Variables}

Neither age nor gender was significantly associated with global cognition. As expected, a greater number of years of formal education were associated with better global cognition (Estimate $=0.06 ; \mathrm{SE}=0.01, P<0.001)$. Compared with Whites, Blacks showed significantly lower global neurocognitive scores (Estimate $=-0.37$; $\mathrm{SE}=0.08, P<0.001$ ). Broadly similar relationships were found for each of the five neurocognitive domains.

\section{Cognitive Activity Frequency and Neurocognitive Function}

In a series of linear regression models, adjusted for demographics (age, sex, education and race), we found that more frequent cognitive activity was associated with better global cognition (Estimate $=.13 ; \mathrm{SE}=.06, P=0.026$ ) and with two of the five neurocognitive domains, semantic memory (Estimate $=.19 ; \mathrm{SE}=.08, P=.017$ ) and perceptual speed (Estimate $=0.28 ; \mathrm{SE}=0.08, P=0.001)$. See Table 2.

The significant association between frequency of cognitive activity and global cognition was essentially unchanged after controlling for employment status, common medical conditions, vascular risk score, HCV serostatus, CD4 nadir, and average number of lifetime drugs used. However, when number of depressive symptoms was entered, the association was reduced to a nonsignificant trend (Estimate $=0.10 ; \mathrm{SE}=0.10, P=0.060$ ). The associations of cognitive activity and the individual domains were essentially unchanged after controlling for all covariates.

\section{Cognitive Activity Frequency and Race Interaction}

The Race $\mathrm{x}$ Cognition interaction term was significant in subsequent models, suggesting that the association of cognitive activity and global neurocognitive function was only apparent among Blacks (Estimate for interaction $=0.38 ; \mathrm{SE}=0.11, P<0.001$ ). After controlling for 
all of the covariates listed above, this association remained significant, with $P$ values ranging from $P<0.001$ to $P=0.01$ (controlling for Hepatitis C). Significant interactions between race and frequency of cognitive activity were also present for episodic memory (Estimate $=$ $0.39 ; \mathrm{SE}=0.14, P=0.006$ ), working memory (Estimate $=0.54 ; \mathrm{SE}=0.19, P=0.005$ ) and perceptual speed (Estimate $=0.41 ; \mathrm{SE}=0.17, P=0.02$ ) after controlling for covariates.

\section{Discussion}

In this study of 176 older persons with HIV we found that, consistent with the aging literature [1, 6-7], cognitive activity was significantly associated with better neurocognitive function. Specifically, more frequent participation in cognitive activity was associated with higher global neurocognitive function, and two of the neurocognitive domains, semantic memory and perceptual speed, but only among Black participants. This relationship between current cognitive activity and neurocognitive function remained significant after controlling for demographic variables (age, sex, education), and important covariates, such as medical conditions, vascular risk score, HCV serostatus, CD4 nadir, and average number of lifetime drugs. Furthermore, general health and HIV disease status were comparable for the two groups. Interestingly, when depressive symptoms were added to the model, the association between cognitive activity and neurocognitive function was reduced to a non-significant trend, suggesting that the association is not completely independent of depressive symptoms.

Overall, these results suggest the association between cognitive activity and neurocognitive function may be stronger in HIV+ Blacks compared with Whites. However, at present the significance of this finding is unclear. Factors that contribute to racial disparities in neurocognitive health among older persons, such as years of education and income [15], were significantly higher among White participants in our study. Additionally we note that Whites reported significantly more reading, museum visits, and attendance at plays or concerts, activities that are most likely more frequent among individuals with higher education and more disposable income.

Our study is limited by the use of a self-report measure of a restricted number of cognitive activities, although the measure has been used in numerous studies of older adults and has been shown to predict neurocognitive decline and risk of Alzheimer's disease [1, 7, 16-18]. Our measure of history of illicit drug was crude and does not take into account patterns of drug use over time. Future studies should use more sensitive measures that incorporate frequencies and patterns of past use. Also, we had a much smaller proportion of Whites among the study sample. Had our study included more Whites, we would have had slightly more power.

Our study is advantageous compared with previous reports from the cognitive activity literature among HIV+ individuals, in that the sample size was larger and neurocognitive testing was more comprehensive. Our study sample characteristics enabled us to observe potentially important demographic factors contributing to disparities in neurocognitive health and thereby help to establish factors associated with better neurocognitive outcomes in persons aging with HIV. An important implication of these findings for HIV+ Blacks and Whites suggest that improved access to cognitive activity interventions, in particular among 
middle to older aged Blacks, could be an important target to reduce late-life disparities in brain health and neurocognitive function.

\section{Acknowledgments}

The authors gratefully thank the CEDHA participants, Tarisha Washington and Virginia Maravillas for study coordination and data collection, the CEDHA staff, the Ruth M. Rothstein staff, and the Rush Alzheimer's Disease Center Staff. We also thank Dr. Eileen Martin in the Department of Psychiatry at Rush University Medical Center for her advice and comments on this version of the manuscript. This research was supported by National Institute on Minority Health and Health Disparities grant (P20MD6886).

This research was supported by National Institute on Aging grant P20MD6886, and a grant from the Illinois Department of Public Health.

All procedures performed in this study with human participants were in accordance with the ethical standards of the institutional review boards of Rush University Medical Center and the Cook County Health and Hospital System and with the 1964 Helsinki declaration and its later amendments. Informed consent was obtained from all individual participants included in the study.

\section{References}

1. Wilson RS, Scherr PA, Schneider JA, Tang Y, Bennett DA. Relation of cognitive activity to risk of developing Alzheimer disease. Neurology. 2007 Jun.:1911-1920. [PubMed: 17596582]

2. Chan JSY, Yan JH, Payne VG. The impact of obesity and exercise on cognitive aging. Front Aging Neurosci. 2013 Dec 20.5:97. [PubMed: 24391586]

3. Wilson RS, Boyle PA, Yu L, et al. Life-span cognitive activity, neuropathologic burden, and cognitive aging. Neurology. 2013 Jul 23; 81(4):314-21. [PubMed: 23825173]

4. Krueger KR, Wilson RS, Kamenetsky JM, Barnes LL, Bienias JL, Bennett DA. Social engagement and cognitive function in old age. Exp Aging Res. 2009 Jan-Mar;35(1):45-60. [PubMed: 19173101]

5. Centers for Disease Control and Prevention. Diagnoses of HIV infection among adults aged 50 years and older in the United States and dependent areas, 2007-2010. HIV Surveillance Supplemental Report. 2013; 18(3) http://www.cdc.gov/hiv/topics/surveillance/resources/reports/\#supplemental Published February 2013. Accessed [September 20, 2015].

6. Wilson RS, Mendes de Leon CF, Barnes LL, et al. Participation in cognitively stimulating activities and risk of incident Alzheimer disease. JAMA. 2002; 287(6):742-748. [PubMed: 11851541]

7. Wilson RS, Barnes LL, Krueger KR, Hoganson G, Bienias JL, Bennett DA. Early and late life cognitive activity and cognitive systems in old age. J Int Neuropsychol Soc. 2005 Jul; 11(4):400-7. [PubMed: 16209420]

8. Fazeli PL, Crowe M, Ross LA, Wadley V, Ball K, Vance DE. Cognitive functioning in adults aging with HIV: A cross-sectional analysis of cognitive subtypes and influential factors. J Clin Res HIV AIDS Prev. 2014 Feb 18; 1(4):155-169. [PubMed: 25386565]

9. Becker JT, Dew MA, Aizenstein HJ, et al. A pilot study of the effects of internet-based cognitive stimulation on neuropsychological function in HIV disease. Disabil Rehabil. 2012; 34(21):1848-52. [PubMed: 22458375]

10. Manly JJ, Smith C, Crystal HA, et al. Relationship of ethnicity, age, education, and reading level to speed and executive function among HIV+ and HIV- women: The WIHS neurocognitive substudy. J Clin Exp Neuropsychol. 2011 Oct; 33(8):853-863. [PubMed: 21950512]

11. D’Agostino RB, Wolf PA, Belanger AJ, Kannel WB. Stroke risk profile: adjustment for antihypertensive medication. The Framingham Study. Stroke. 1994; 25:40-43. [PubMed: 8266381]

12. Boyle PA, Wilson RS, Aggarwal NT, et al. Parkinsonian signs in mild cognitive impairment. Neurology. 2005; 65:1901-1906. [PubMed: 16380610]

13. Kohout FJ, Berkman LF, Evans DA, Cornoni-Huntley J. Two shorter forms of the CES-D (Center for epidemiological studies depression) depression symptoms index. J Aging Health. 1993 May; 5(2):179-93. [PubMed: 10125443] 
14. SAS Institute Inc. SAS/STAT User's Guide, Version 9.3. SAS Institute; Cary, NC: 2011.

15. Sisco S, Gross A, Shih R, et al. The role of early-life educational quality and literacy in explaining racial disparities in cognition in late life. J Gerontol B Psychol Sci Soc Sci. 2015 Jul; 70(4):55767. [PubMed: 24584038]

16. Wilson RS, Segawa E, Boyle PA, Bennett DA. Influence of late-life cognitive activity on cognitive health. Neurology. 2012; 78(15):1123-9. [PubMed: 22491864]

17. Barnes LL, Wilson RS, de Leon CF, Bennett DA. The relation of lifetime cognitive activity and lifetime access to resources to late-life cognitive function in older African Americans.

Neuropsychol Dev Cogn B Aging Neuropsychol Cogn. 2006 Sep-Dec;13(3-4):516-28. [PubMed: 16887787]

18. Marquine MJ, Segawa E, Wilson RS, Bennett DA, Barnes LL. Association between cognitive activity and cognitive function in older Hispanics. J Int Neuropsychol Soc. 2012 Nov; 18(6):104151. [PubMed: 22676914] 


\section{Table 1}

Demographic Characteristics by Race

\begin{tabular}{lllll}
\hline & $\begin{array}{l}\text { Black } \\
\mathbf{N}=\mathbf{1 2 4}\end{array}$ & $\begin{array}{l}\text { White } \\
\mathbf{N}=\mathbf{5 3}\end{array}$ & $\begin{array}{l}\text { Total } \\
\mathbf{N}=\mathbf{1 7 7}\end{array}$ & Group Comparison \\
\hline Characteristic & $\boldsymbol{M}(\mathbf{S D}) / \mathbf{N}(\%)$ & $\boldsymbol{M}(\mathbf{S D}) / \%$ & $\boldsymbol{M}(\boldsymbol{S D}) / \%$ & $\begin{array}{l}\text { t-test } / \chi^{2} \\
\boldsymbol{P} \text { value }\end{array}$ \\
Age & $59.3(5.5)$ & $57.4(5.2)$ & $58.7(5.5)$ & 0.65 \\
Education in years & $12.6(2.4)$ & $14.6(3.3)$ & $13.2(2.8)$ & $<0.01^{*}$ \\
Women & $42(17.5 \%)$ & $6.2 \%$ & $24 \%$ & 0.53 \\
Income < \$25,000 & $81 \%$ & $49 \%$ & $71 \%$ & $<0.01 *$ \\
Currently employed & $26(21 \%)$ & $26(49 \%)$ & & $<0.001$ \\
CD4 nadir & $252(177)$ & $278(162)$ & $260(172)$ & 0.55 \\
Average No. of illicit drugs used - lifetime & $2.1(2.0)$ & $1.8(1.5)$ & $2.18(1.9)$ & 0.04 \\
Medical conditions & $2.9(1.6)$ & $2.9(1.4)$ & $2.9(1.6)$ & 0.35 \\
Vascular risk & $1.5(0.7)$ & $1.3(0.8)$ & $1.5(0.74)$ & 0.11 \\
Depressive symptoms & $2.6(2.6)$ & $2.8(2.3)$ & $2.6(2.5)$ & 0.31 \\
MMSE score & $28.2(1.3)$ & $28.9(1.3)$ & $28.4(1.3)$ & 0.64 \\
\hline
\end{tabular}

* Statistically significant at the $P<0.05$. 


\section{Table 2}

Relation of cognitive activity to current cognitive function

\begin{tabular}{l|l|l|l}
\hline Cognitive function & Estimate & $\boldsymbol{S E}$ & $\boldsymbol{P}$ \\
\hline Global Cognition & .13 & .06 & .026 \\
\hline Episodic memory & .03 & .07 & .625 \\
\hline Semantic memory & .19 & .08 & .017 \\
\hline Working memory & .10 & .10 & .289 \\
\hline Perceptual speed & .28 & .08 & .001 \\
\hline Visuospatial ability & .07 & .09 & .423 \\
\hline
\end{tabular}

Note: Results were based on linear regression models that controlled for age, sex, education, and race. 